\title{
Reason Maintenance in Product Modelling via Open Source CAD System
}

\author{
Z. Ibrahim ${ }^{\#}$, S. B. Mohamed", M. Minhat ${ }^{\# \#, ~ A . ~ S . ~ M o h a m e d ~}{ }^{\# \#, ~ M . ~ R . ~ M u s a n i h ", ~ Z . ~ A b d ~ R a h m a n ", ~}$ \\ Zairi Ismael Rizman ${ }^{* * *}$ \\ ${ }^{\#}$ Faculty of Innovative Design and Technology, Universiti Sultan Zainal Abidin, Terengganu, 21300, Malaysia \\ E-mail:zibra@unisza.edu.my \\ ${ }^{\#}$ Faculty of Manufacturing Engineering, Universiti Teknikal Malaysia Melaka, Melaka, 76100, Malaysia \\ E-mail:mohdm@utem.edu.my \\ *East Coast Environmental Research Institute, Universiti Sultan Zainal Abidin, Terengganu, 21300, Malaysia \\ E-mail: saifulbahri@unisza.edu.my \\ ${ }^{* *}$ Faculty of Electrical Engineering, Universiti Teknologi MARA, Dungun, Terengganu, 23000, Malaysia \\ E-mail:zairi576@tganu.uitm.edu.my
}

\begin{abstract}
The present and future challenges of a new product design, forecasting and risk management launch strategy for a new product modelling decision process. This paper intends to propose and to look towards the development of a low-cost integrated CAD-CAPP-CAD/CAM product modelling system for the design and manufacture of a proposed product. It is a mapping between several design phases like functional design, technical design and physical design. The modelling data generation process begins with the drafting of a product to be maintained using the drafting software package. From the CAD drawing, the data are transferred to be used as the product models and a CAPP software package will then prepare the operational parameters for the manufacturing of the product. These process data are relayed to a CAM software package, which will then generate the automating informationprocessing functions. The final stage of the function is to support design and manufacturing operations that may have reaped many benefits in terms of its initial equipment and software costs.
\end{abstract}

Keywords — reason maintenance; product modelling; modular design; open sources CAD

\section{INTRODUCTION}

Over five decades ago, manufacturing industries have been looking for more efficient processes to manufacture products through Computer Numerical Control (CNC) machines [1], [2], [3]. CNC manufacturing has since relied on data and product modeling and therefore technologies related to Computer Aided Design (CAD), Computer Aided Engineering (CAE), Computer Aided Process Planning (CAPP) and Computer Aided Manufacturing (CAM) are primarily important. The development of a product modeling consists of three elements which are firstly a problem definition, secondly a product modelling involving synthesis, a rough physical implementation of the product model as well as evaluation of the model functional behavior and finally, the selection of the best product model [4].

The information, database and data model that $\mathrm{CNC}$ manufacturing processes encompass may be divided into product and process data. These data are often exchanged between various manufacturing facilities in today's global economy [5]. The industry is categorized by an increasing demand for diversity and increasing demands on product development time because of an increasing customer needs and decreasing of product life-cycle and time-to-market. These trends force manufacturing to focus on the longpending product development planning to adapt various market structures and short product life-cycle [6].

The proposed product development reflects an overall procedure of product structuring for customers, assembly, and maintenance [5]. The product model can be expressed as a mapping between several design phases like a functional design, technical design, and physical design. Namely, the product modeling process starts with the identification of customer needs and formulation them as functional requirements. These requirements are then mapped onto the physical domain by identifying the design parameters. 
Product modeling system for the design and manufacture of any proposed product consists of a mapping strategy between several design phases like functional design, technical design and physical design as shown in Fig. 1. In the design phases, the main objectives and all of the project research activities or tasks are shown. It also elaborates and illustrates the critical activities of each variety for each phase.

The system shall define the product, customer, functional and structural variety. A product variety is defined as the diversity of products, three types of variety which is customer, functional and structural variety [7]. A customer variety has the capability to provide a differentiation of customer requirements. A functional variety is used to mean any differentiation in the attributes related to product functionality and finally, a structural variety has a common relationship to part and sub-assembly variation.

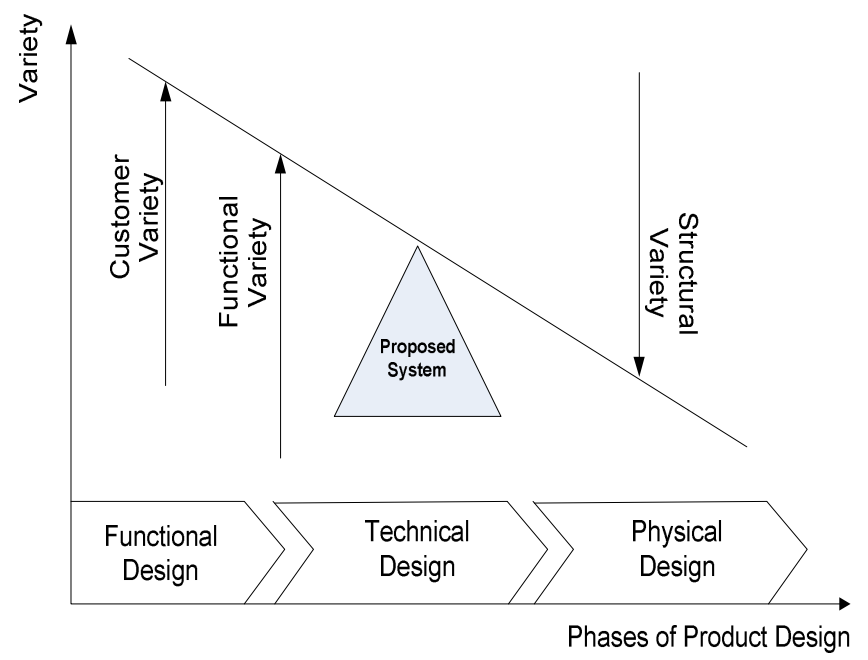

Fig. 1 The design phases

There are several disadvantages of using CAD software package that had been divided into two main components that are cost and error. As with any business, costs are always a factor. Cost quite a lot more than conventional machinery by using CNC machines, it also does not eliminate the need for expensive tools. Moreover, parts such an example tooling and machines are costly and their purchases needed extensive justification and are expensive for maintenance. For errors, CNC machines do not totally eliminate errors. Make incorrect alignments, fail to locate parts properly on a jig and operators can still push the wrong buttons.

To overcome this problem, virtual CAD simulator has been promoted because it is a software to illustrate, verify and simulate the operation of a CNC machine tool's PC. The operation of the task is displaying an image of the threedimensional model of the machine by animation from the simulation device that had received historical data by lapsing time information and positional information associated with the lapsing time information of the operation [7]. A virtual CAD simulator helps to detect the error occur and the improvement can be done.

The benefit of using this simulation is helping to increase the preparedness of users, reduce the occurrences of programming errors and machine crashes and improve the efficiency of time spent in the laboratory [9]. Simulation techniques are becoming increasingly useful to help industries better manage their resources and not just for the educational benefit of the students. A method is provided for virtual machining which helps allocates errors in the design and manufacture of machine tools, diagnose the source of errors in machine tools and predict the accuracy of parts to be made on machine tools [10], [11]. The machining process and workpiece forming process can be observed directly in the virtual environment, so the correctness of the CAD/CAM code can be checked in advance to avoid the interference, collisions or other problems in the actual lathe processing and reduce the scrap rate of the workpiece and tool in the actual machining process.

The main objective of this research project is to develop a systems methodology on how to maintain the product modeling for the line-up options. The project research outcome aims of the software in spite of the available options hardware techniques. The specific objective of the project research can be achieved through a prototype product model which represents the best solution for model selection due to give the reasoning algorithm.

A new set of product model specifications, standards, appropriate chart, user interfaces, and languages are to be used. The software will be used to give the algorithm a distributed, simulation and real-time product model selection characteristics. The scope of this research project is defined in accordance with the integrated CAD-CAPP-CAD/CAM product modelling system that the prototype will deal with, that is the modelling selection operations by agreeing that the product modelling is the heart of modern design tools and traditionally and they are done in an interactive vendorspecific proprietorship, manually or by a software. It has been a bottleneck for supporting interactive and interoperabe multi modular product modelling. Traditional product model ling also closed and inflexible in nature. Because of this, research has been ongoing in incorporating from traditional product modelling with the development of an interactive open source CAD system.

\section{MATERIAL AND METHOD}

\section{A. Project Tasks Product Modelling}

The focus of this research project is maintaining the product model in searching for the best product model which can satisfy the initial functional requirements. A design solution meets a number of constraints such as cost, weight, features, and shapes but the most important is the capability of performing the function for which the product is aimed. Usually, a number of product models satisfy functional requirements and should be considered as candidate design solutions.

CAD systems sit at the beginning of the process starting from product design using $\mathrm{CAD}$ tools for the generation of machining instructions that instruct a $\mathrm{CNC}$ machine to produce the final product. This process chain also includes process planning and machining. Traditional product modeling, in conjunction with CAD systems, has made it possible to produce goods with consistent quality and enabled the industry to enhance productivity with a high degree of flexibility in a manufacturing system. Traditional product modeling alternatively offers an open source CAD 
system architecture for industrial and integration to other systems. Despite the short history of traditional product modeling and open source CAD system, considerable research has already been carried out both internationally and within countries/organizations. This has involved the participation of universities, industries and CAD systems manufacturers in numerous efforts, especially concerning a new system framework for open source CAD system.

The problem is to develop an efficient search procedure to identify all feasible product models, that is product models that provide accomplishment of the overall design function by properly defining relationships among the design subfunctions. There is no feasible model should be overlooked since nobody knows which functionally valid model will lead to the best design solution subject to other design requirements and objectives.

The project research work starts with important activities involved in product modeling. Fig. 2 explains that the project research will go through the following three phases, first is the development of a model base of abstract components that correspond to design functions.

Secondly, the synthesis of the abstract product models is undergoing. Finally, the analysis of the functional behavior of product concepts based on the abstract models is to be done. It correlates with building a conceptual framework, based on which a testbed will be developed for the system. The project research involves working with a generic and native program, thus enabling the system to have the desired intelligence and operability.

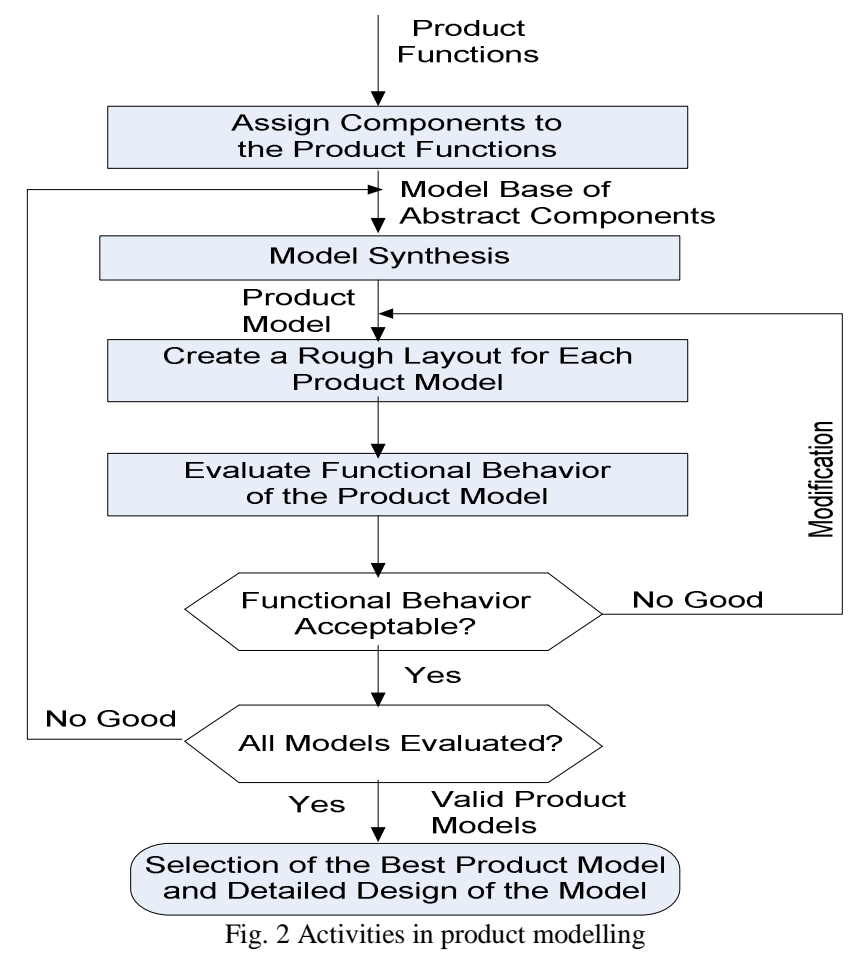

\section{B. Modules in The Systems}

As stated earlier, product model must satisfy both structural and functional requirements which are going through processes of synthesis of abstract components and analysis of functional behavior. The best overall product model will be resulted in the detailed design of the product.
In the future, for a new product modeling, it will consider the technology change and cycle time as well as engineering to manufacturing and marketing links. Recently, globalization, the world wide web, and the internet have been determinants for future new product modeling. In technological change and cycle time issue, the need to get to market fast will become even more important as rapid technological change and the intensity of competition increase. Industries will be utilizing more overlap in their processes and the traditional sequential process will become more ad hoc and iterative.

Engineering, manufacturing and marketing links will practice a conjoint analysis and quality functional deployment supply initial approaches to linking engineering design to market responses. It has become to make another step forward. The detailed engineering design activities need to be integrally linked to markets through micro design/manufacturing specifications.

Globalization affects the world and the marketplace. Increasingly firms are considering global products that reach economically and psychologically specified segments rather than geographically defined country market segments. The manufacturer assesses a global market that simultaneously exists in 50 countries that span from China, Poland, France, Canada, Kenya, Chile, and Japan [12]. The world wide web represents a major new technology for marketing. This paradigm shift will present opportunities and challenges for new product development. In the future, web shopping for both consumer and industrial products and services will become common in our markets.

Recently, the CAD system with an open source solid modeling computer-aided design system with a complete Integrated Development Environment (IDE) is well-known for creating interactive 3D graphics as well as a suite of editing tools. It is the first product to offer a complete range of tools in a single IDE. Researchers need to invest the time to understand the design model, a key artifact of the appropriate applications that are not part of an IDE. The operators and managers have to check the source code for compliance with established standards for CAD services in assisting the computerized design.

For information, computer applications for product modeling are born to make product design and production more efficient. The early programs soon became complex systems, expanding their functionality to all aspects of the product life cycle. This project research reviews the key features of a class of those toolkits, the integrated development environments: intuitive programming language, insulation from mainstream system changes, reduced maintenance expense and rapid response to evolving requirements.

IDE concept all is in the name to integrate and it forms an integral part of a system, with which it interacts and share resources. Development serves to create new applications, bringing the capabilities or possibilities of the mainstream system to a more effective state in which the environment is a well-configured, comprehensive set of tools and resources conceived for creating professional extensions of the system. 


\section{Component-Products Function}

The first task is to identify of Product-Process Model and Parameters so that Component-Products Function MODULE can be completed. The product model same example will be used as for the concept approval stage. The main module to be developed is a model base of abstract components that correspond to design functions.

Designing a product becomes more common due to the rapid development of technology in the manufacturing industry with a large number of highly skilled draughtsman are required. The most skilled of the technician must be strengthened based on applied knowledge because the characteristic of design techniques is highly required knowledge and difficult to program and operate.

It also requires periodic setup for product changes and setup performed in-line with the tool to live by running product take away from production time and may be classified as waste. An important aspect in conducting a design activity is the product modelling stage that including requires a skillful designer and an appropriate design modelling media.

\section{Model Synthesizer}

The Model Synthesizer MODULE in the next stage consists of the development of research methodology and the synthesis of the abstract product models. This is also known as the generic data model program. Recent advances in product modelling are the results of responding to the customer's requirements. The new traditional methods, software, and hardware available in the market make it possible to graphically simulate the part features modelling and to use an open source component for further improvement of manufacturing processes.

The module presents the main results of the knowledge and content structuring via methods of collaborative ontology design project, which aims to develop a novel paradigm for knowledge structuring based on the interplay between cognitive psychology and ontology engineering. By the conceptual model, it defines the main domain concepts and relations between them in the form of a graph, map or diagram. This approach considers individual cognitive styles and uses recent advances in knowledge engineering and conceptual structuring. It aims to create new, consistent and structurally holistic knowledge bases for various areas of science and technology.

\section{E. Model Layout}

The next phase is the Model Layout MODULE and the modules will take several months to complete. The module builds a conceptual framework, based on which a testbed will be developed. In this module, it is crucial that software quality is an important issue in software engineering. The evaluation of software architecture is to achieve quality scenarios, which reduces development and maintenance costs. This work presents a discrete event simulation environment for the software architecture assessment considering both functional and quality aspects. Discrete Event System Specification (DEVS) formalism and the underlying framework are used to specify the simulation elements.
This module is concerned with the simplification of discrete event simulation models. Specifically, the necessary concepts and tools are investigated for the automatic generation of simplified versions of a discrete event model. The simplification process developed utilizes observations of model behavior. Therefore, the simplified models that are generated adhere to all the canons of this formalism. The implementation is designed as an extension to DEVSScheme, a hierarchical modular discrete event modelling and simulation environment. It consists of two layers namely the lower layer observes the model behavior and the higher layer generates the code from the observation data obtained at the lower layer. The resulting system allows one to generate simplified models at any level of the model structure hierarchy. A local area network model base is developed and used to demonstrate the validity and complexity reduction of the simplified models relative to the models from which they are extracted.

\section{F. Model Layout}

The Functional Behavior MODULE consists of a Construction of Functional and Behavior Operator and the analysis of the functional behavior of product concepts based on the abstract models. The final phase is a simulation, pilot trial, data gathering, validation and thesis writing.

Conceptual design defines the basic form and function of a product. In this project research, the use of functional 3D models conceptual design is developed. The researcher performs conceptual design by first defining how the product can be used in the form of a product use model, followed by creating basic 3D models using the bare hands in an Augmented Reality CAD Environment (ARCADE). A multilevel function-behavior-structure modelling framework is developed for design reasoning and evaluation to ensure that the design is functional and geometrically consistent. The functional behavior of a product can be simulated as a prototype in ARCADE to demonstrate the use of the product and detect potential usability issues. Two case studies of the design of a tabletop cleaner and a fruit processor are conducted to demonstrate the application of functional 3D models in conceptual design.

Features illustrate CAD concepts with respect to engineering problems rather than to a particular syntax includes practical information on automation through an open source CAD system in the design process and animation. Open source CAD system explains the mathematical tools used in related operations and takes a self-directed approach that is not based on particular CAD software as well as a covers concept generation, approaching design and modelling supported by practical examples and downloadable demo videos.

\section{G. Standard for Product Modelling Protocol}

Product modeling is at the center of various new product development paradigms designed to meet this challenge. Therefore, it has received major attention from application and research communities. The engineering professional is to develop new products that have the short lead-time, highest quality and lowest cost with optimal life-cycle consideration. The scopes and approaches of product modeling have evolved rapidly in recent years. In this way, 
the computer controls the design and manufacturing of the model. Typically, CAD software uses a wide scope and approaches of product modeling have evolved rapidly in recent years.

In today's environment, the product modeling data are managed in many different systems, often with little integration and with a great deal of data redundancy. The recent product model (ISO10303) is addressing the data exchange flow problem by offering a unified standard to describe all the aspects of a product during its life-cycle. This endeavor led to the establishment of the future product model STEP [ISO 10303-1, 1994]. The unique feature of the model is that it integrates product data by providing a single product data standard. The protocol does so through establishing various Application Protocols (APs) targeted at different application domains, be it design, manufacturing, or maintenance. It provides both broadly useful data modeling methods and data models focused on specific industrial uses.

Important parts in CAD system are product modeling protocol, research tools of IDE-compliant CAD options and the best selected system fits. In order to promote CAD system validity, it is particularly important to enhance the interface and any icon toolbar involved. The relationship between the data model and the features are obtained through part design stage, simulating, some reliable theoretical basis can be achieved in improving and enhancing CAD system performance.

Besides that, other issues that make this research is undertaken is to provide an alternative to local universities which always incurred any cost of CAD system despite the open source CAD system is available and downloaded freely on any network. Some of those open source CAD systems provides typical performance and quality as well as knowledge based compared to commercial CAD system package. The expected outcome of this research is to utilize the best selected open source CAD system that has been provided on the internet to the industries and higher educational institutions.

An IDE is a software application that provides comprehensive facilities to computer programmers for software development. An IDE normally consists of a source code editor, build automation tools and a debugger. Most modern IDEs have intelligent code completion.

IDEs initially become possible when developing via a console or terminal. Early systems cannot support one, since programs are prepared using flowcharts, entering programs with punched cards (or paper tape, etc.) before submitting them to a compiler.

\section{RESULTS AND DISCUSSION}

\section{A. Application Protocol}

An application protocol focuses on a particular application domain. This project presents a framework, a tool designed to support every step of a security protocol's life cycle. Its IDE eases the task of protocol design, debugging and simulation.

Security protocols describe a strategy that two or more parties can follow to obtain certain guarantees about each other by exchanging messages over an untrusted medium.
For instance, parties may wish to ascertain each other's identity, exchange secret information or even communicate without revealing their identity.

Experience shows that the design and implementation of security protocols are a complex and error-prone task. General purposes programming languages and environments provide little help. A protocol that specified in a few lines yields thousands of lines of code in languages such as Java and $\mathrm{C}++$. As the semantics of the protocol become unclear, debugging can turn into a long and painful activity.

\section{B. Open Sources CAD Systems}

Open-source is denoting software for which the original source code is made freely available and may be redistributed and modified. The term "open source" software is used by some people to mean more or less the same category as free software. It is not exactly the same class of software that accepts some licenses that to be considered too restrictive, and there are free software licenses that have not accepted. However, the differences in extension of the category are small which is nearly all free software is open source, and nearly all open source software is free.

Generically, open source refers to a program in which the source code is available to the general public for use and/or modification from its original design free of charge that is open. Open source code is typically created as a collaborative effort in which programmers improve upon the code and share the changes within the community. Open source sprouted in the technological community as a response to proprietary software owned by corporations.

A certification standard issued by the Open Source Initiative (OSI) that indicates that the source code of a computer program is made available free of charge to the general public. The rationale for this movement is that a larger group of programmers not concerned with proprietary ownership or financial gain will produce a more useful and bug -free product for everyone to use. The concept relies on peer review to find and eliminate bugs in the program code, a process which commercially developed and packaged programs do not utilize. Programmers on the Internet read, redistribute and modify the source code which forcing an expedient evolution of the product. The process of eliminating bugs and improving the software happens at a much quicker rate than through the traditional development channels of commercial software as the information is shared throughout the open source community. It does not originate and channel through a corporation's research and development cogs.

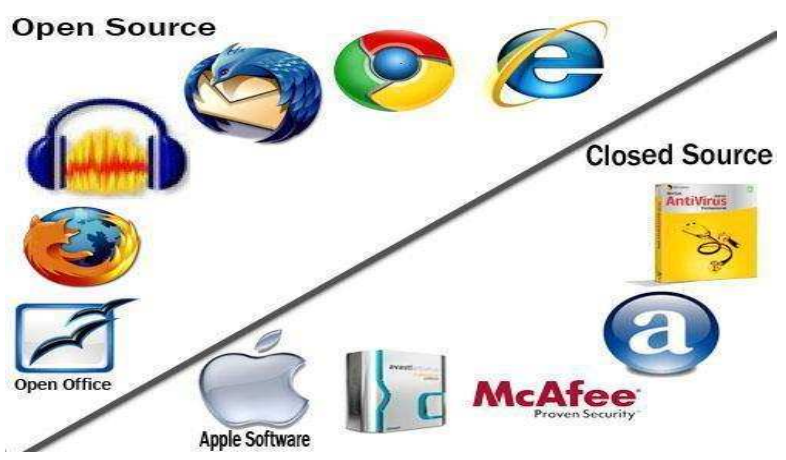

Fig. 3 Differences between closed source software and open source software 
According to the project research title, the medium to find the open sources CAD systems is by using the open source software itself. It is software for which the original source code is made freely available and it may be modified and redistributed. Most open source software is also distributed as free with limited restrictions on how it can be used, hence the term "free" when used to describe open source carries two meanings that are free of cost and free to do with the software as you wish. Fig. 3 shows that the differences between closed source software and open source software.

\section{Open Cascade}

Open Cascade Technology (OCCT), formerly called CAS.CADE, is an open source software development platform for 3D CAD, CAM, CAE etc. that is developed and supported by Open Cascade SAS. CASCADE (abbreviated from Computer Aided Software for Computer Aided Design and Engineering) is originally developed in the early 1990s by Matra Datavision, developer of Euclid CAD software as the underlying infrastructure for its future version Euclid Quantum. In 1998, the company abandoned software development to concentrate on services, and most of the software development facilities are sold to Dassault Systems, developer of competing CATIA.

Open CASCADE is a software development kit (SDK) intended for development of applications dealing with 3D $\mathrm{CAD}$ data that freely available in open source. It includes a set of $\mathrm{C}++$ class libraries providing services for $3 \mathrm{D}$ surface and data exchange, solid modelling, rapid application development and visualization.

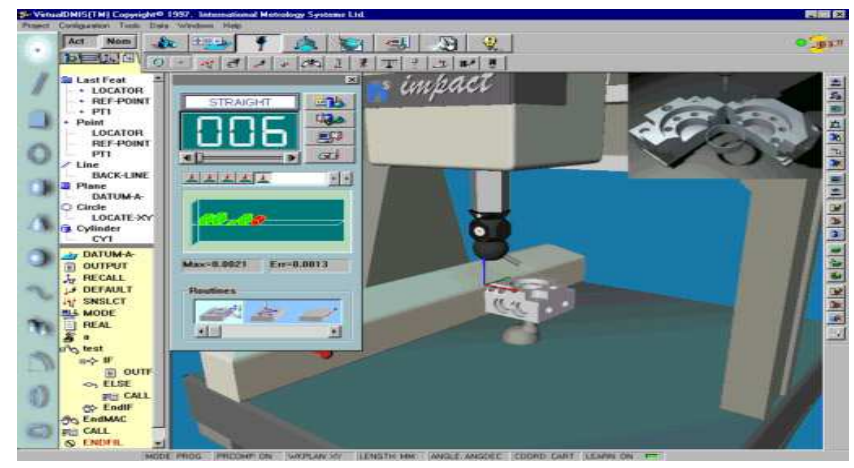

Fig. 4 Open CASCADE software

It is best applied in the development of specialized CAD/CAM/CAE applications. Pre and post-processor for finite element analysis software, numerical simulation programs, and CNC/CAM path generators are the typical applications built with the help of Open CASCADE Technology. Fig. 4 shows that the process of Open CASCADE software with 3D visual and detail information of icon that has provided.

\section{Expected Outcomes}

Open source CAD systems have become popular in the manufacturing sector in the last decade. The primary cost is expensive to afford all aspects related to the operations of the CAD project. Moreover, an important aspect in conducting open source CAD system is the programming stage that including requires a skillful programmer and an appropriate programming media. The cost to hire CAD specialist is too expensive. It is because of the lack of specialist in this area and makes it possible for people who expert in the CAD area is too demanding for the best salary.

An important part of CAD systems is a data exchange system. In order to promote CAD design precision, it is particularly important to enhance the control method's research of open source CAD system. The relationship between the system's parameters and the performance of data exchange are obtained through simulating, some reliable theoretical basis can be achieved in improving and in the future which enhancing open source CAD system performance.

The precision and accuracy of CAD systems directly affect the dimensional accuracy of designing parts. This means precision is very important to ensure the accuracy of the design during the future process. So, to ensure the accuracy, installation and tutorial setup must in properly setting and followed the specified dimension of the part data design.

Besides that, other issues that make this project is viable are the local university always want to simulate the virtual $\mathrm{CAD} / \mathrm{CAM} / \mathrm{CNC}$ simulator. Whereas, these virtual $\mathrm{CAD} / \mathrm{CAM} / \mathrm{CNC}$ simulator has been provided freely by open source software. Some of these simulators provided the same performance and quality as commercial virtual $\mathrm{CNC}$ simulators. The expected outcome is to utilize the virtual CAD simulator that has been provided in the open source software to the industries and higher educational institutions.

\section{CONCLUSIONS}

Product modeling by software implementation method requires researcher to key in input data for the models and implementing the process in the proposed system. All the models shall match the needs requirement based on the theory function. By using the power of the existing empirical data, the researcher enables to do programmatic project research and the user of the system may have the great designers or co-authors to undergo the generalizability process in which a statistical framework is run for conceptualizing, investigating, and design any reliable observations and finally a solution.

The project review is to be completed regarding product modeling, design maintenance system and technologies as well as suitable system software tools. Also to be reviewed is the literature of any availability of standard documentation related to the proposed system. Early research publications conclude that one of them shall take a holistic view of these new product modelling technologies and attempted systems to arrive at complete definitions of this product modelling technologies. Implementations are to be reviewed to showcase some of these technologies.

The project research shall conclude that the proposed system and reason maintenance in product modelling are two emerging requirements that may hold the key to developing a new generation of product modeling system that is reconfigurable and modularized in structure, adaptable and interoperable in functions. Three major parts of the current functional design, technical design, and physical design are presented. It is also included in this paper where the system will highlight some of the key issues with relevant facts. 


\section{ACKNOWLEDGMENT}

We are grateful for the UniSZA and UTeM for the facilities and research fund provided.

\section{REFERENCES}

[1] C. Cunha, B. Agard and A. Kusiak, "Design for cost: Module-based mass customization," IEEE Transactions in Automation Science and Engineering, vol. 4, pp. 350-359, Jul. 2007.

[2] M. Minhat, S. B. Mohamed, M. S. Kasim, M. J. Kadhim, M. A. Sulaiman and R. Besar, "Retrofitting a conventional lathe to a digital intelligent system," in Proc. AICS'14, 2014, p. 167-174.

[3] S. B. Mohamed, A. Jamaeel and M. Minhat, "A review on intelligence STEP NC data model and function block CNC machining protocol," Advanced Materiasl Research, vol. 845, pp. 779-785, 2014.

[4] R. Vujosevic, A. Kusiak and E. Szczerbicki, "Reason maintenance in product modeling," Journal of Engineering Industry, vol. 117, pp. 223-231, May 1995.

[5] L. U. Glen and R. H. John, Design and Marketing of New Products. 2nd ed., New Jersey, USA: Prentice-Hall International Inc, 1993.

[6] T. R. Browning, E. Frikie and H. Negele, "Key concept in modeling product development processes," Systems Engineering, vol. 9, pp. 104-128, Jun. 2006.
[7] J. R. Cho and H. S. Mok, "Product structuring for customer, assembly and maintenance," M. Eng. thesis, Pusan National University, Busan, Soth Korea, 2000.

[8] A. Watanabe, Y. Nagatsuka and K. Kuribayashi, "Simulation device," Patent No. US 7194396 B2, Mar. 20, 2007.

[9] D. Y. Hoi and X. Huang, "Cutter/workpiece engagement feature extraction from solid models for end milling," Journal of Manufacturing Science and Engineering, vol. 128, pp. 249-260, Feb. 2006.

[10] Y. Altintas, "Virtual high performance machining," Procedia CIRP, vol. 46, pp. 372-378, Dec. 2016.

[11] M. Soori, B. Arezoo and M. Habibi, "Tool deflection error of threeaxis computer numerical control milling machines, monitoring and minimizing by a virtual machining system," Journal of Manufacturing Science and Engineering, vol. 138, pp. 081005-1 081005-11, Aug. 2016.

[12] M. Palmié, M. Zeschky, S. Winterhalter, P. W. Sauter, N. Haefner and O. Gassmann, "Coordination mechanisms for international innovation in SMEs: Effects on time-to-market and R\&D task complexity as a moderator," Small Business Economics, vol. 46, pp. 273-294, Feb. 2016 